\title{
Les Cavaliers du Bouc : brigandage et répression en Basse Meuse (1730-1778)
}

\section{Anton Blok}

\section{Q OpenEdition}

1 Journals

Édition électronique

URL : http://journals.openedition.org/conflits/499

DOI : $10.4000 /$ conflits.499

ISSN : 1777-5345

Éditeur :

CCLS - Centre d'études sur les conflits lilberté et sécurité, L'Harmattan

Édition imprimée

Date de publication : 15 mai 1997

ISSN : 1157-996X

Référence électronique

Anton Blok, "Les Cavaliers du Bouc : brigandage et répression en Basse Meuse (1730-1778) »,

Cultures \& Conflits [En ligne], 24-25 | hiver 1996- printemps 1997, mis en ligne le 27 mars 2007,

consulté le 30 mars 2021. URL : http://journals.openedition.org/conflits/499 ; DOI : https://doi.org/

$10.4000 /$ conflits.499

Ce document a été généré automatiquement le 30 mars 2021.

Creative Commons License 


\title{
Les Cavaliers du Bouc : brigandage et répression en Basse Meuse (1730-1778)
}

\author{
Anton Blok
}

\begin{abstract}
The strenght of collective biography is not in supplying alternative explanations, but in specifying what is to be explained. Historians who have specified what is to be explained via

collective biography often find themselves turning to explanations stressing the immediate setting and organization of everyday life, or relying on something vaguely called " culture". That moves them back to anthropology. Charles
\end{abstract}

Tilly ${ }^{1}$

1 Le banditisme serait " la forme de rébellion la plus aisée, car la plus difficile à réduire pour les Etats "2. Ainsi, les Cavaliers du Bouc, comme on les a appelés, ont sévi à trois reprises (entre 1730 et 1774) dans l'hinterland de Maastricht, la zone frontière entre la République hollandaise et les Pays-Bas autrichiens. Il fallut plus de quarante ans aux autorités hollandaises et autrichiennes pour venir à bout de cette forme de banditisme qui survécut facilement à deux campagnes de répression. Si on ne dispose pas de chiffres précis sur la composition de ces bandes qui, au fil des ans, ont mis à sac plusieurs dizaines d'églises et des fermes, on sait toutefois qu'environ six cents personnes ont été jugées par des tribunaux locaux pour leur appartenance à celles-ci³

On discutera ici du développement de deux formes de violence collective : le banditisme et sa répression. Celles-ci se comprennent en relation avec la culture et l'identité, particulièrement les représentations de l'honneur et de l'infamie, mais aussi les valeurs affectives de la parenté, de la classe, du lieu de résidence et de travail. En premier lieu, nous verrons que les métiers pratiqués au départ par les bandits, leur structure de parenté et leur lieu d'origine, ont fortement favorisé le développement du brigandage 
en Basse Meuse et ont permis sa pérennité. En second lieu, l'histoire politique et militaire de cette région est fondamentale pour comprendre plusieurs aspects, notamment l'aspect chronologique, du phénomène des Cavaliers du Bouc. Les bandes n'apparurent pas avant le début des années 1730 et dans une zone périphérique caractérisée par un important degré de fragmentation territoriale, ce qui soulève un troisième problème : les moyens que les autorités locales pouvaient déployer pour contrôler ces bandes. Quatrièmement, dans les études sur la violence collective populaire, la question des " revendications " apparaît généralement au premier plan. Les revendications peuvent être évidentes lorsqu'il s'agit de révoltes contre les impôts, d'émeutes contre la conscription, et autres exemples similaires de réactions populaires. Cependant, dans le cas du brigandage organisé, elles peuvent être moins claires, et parfois même totalement rhétoriques. Les revendications, s'il y en a, sont souvent vagues, diffuses et ambiguës, donc difficiles à repérer. Cela pourrait en partie expliquer pourquoi le banditisme, lui-même souvent insaisissable, diffus et sporadique, n'a pas de place marquante dans les études sur la violence collective. Mais, avant de traiter de ces questions, nous devons examiner le contexte dans lequel ces bandes se constituèrent.

La Région

3 Les Cavaliers du Bouc ont été actifs en Basse Meuse dans la zone rurale délimitée par les villes de Maastricht, Aix-la-Chapelle, Gulick et Rurmond. Du XVIème siècle jusqu'au début du XIXème siècle, cette région faisait partie d'une zone frontière militaire plus vaste, avec Maastricht comme place-forte et ville de garnison et Liège comme centre important de l'industrie de l'armement. La Basse Meuse était à la fois le carrefour des grandes voies de communications (est-ouest et nord-sud), la zone de contact entre les sphères d'influence des grandes puissances européennes et, ce faisant, parfois leur terrain d'affrontement. La France, l'Espagne, la République de Hollande, l'Autriche et, plus tard, la Prusse, se disputaient en effet la souveraineté sur cette partie de l'Europe. Jusqu'au début du XIXème siècle, cette région eut à souffrir de fréquentes opérations militaires et des partages territoriaux qui en découlaient, tout particulièrement de la division, en 1662, de ce qu'on a appelé les Landen van Overmaas entre la République de Hollande et l'Espagne. Comprenant des territoires espagnols (devenus autrichiens après 1713) et hollandais, ainsi que des sections du duché de Gulick et quelques seigneuries semi-autonomes, cette région était à beaucoup d'égards une zone frontière par excellence ${ }^{4}$. Un historien militaire a soutenu que les Pays-Bas méridionaux jouèrent un rôle important dans le maintien de l'équilibre politique en Europe occidentale, il écrit à ce propos : " Traversé de nombreuses voies d'eau, idéales pour les communications militaires et les transports, ce pays, dense et plat, avait des frontières communes avec l'Allemagne, la France et la République de Hollande. Il était proche de l'Angleterre et couvert de villes et de forteresses. Les Pays-Bas du Sud fournissaient un merveilleux terrain de manœuvre pour les armées européennes, une région où les villes et des parcelles de territoire pouvaient changer de mains lors de traités de paix sans mettre en danger la sécurité générale du continent. Si les Pays-Bas du Sud n'avaient pas existé, il eut été essentiel de les inventer. Des pays se battaient entre eux pour s'emparer d'une ville importante ou d'une étendue de terre qui pourraient servir de monnaie d'échange lors de discussions diplomatiques. Maurice de Saxe conquit Maastricht en 1748, non parce que Louis XV la voulait pour la France, mais pour qu'il put l'échanger contre certaines concessions des Hollandais et de leurs alliés "5.

Outre les frontières politiques, différentes juridictions traversaient cette région, sans compter la séparation entre les Protestants et les Catholiques romains - résultat de la 
conversion au protestantisme imposée (avec relativement peu de succès) par les Hollandais sur leur territoires. Le caractère frontalier de la région était encore renforcé par sa localisation sur des carrefours commerciaux et militaires. Située dans une zone d'interaction européenne majeure, la Basse Meuse reliait les Flandres aux pays Rhénans et à la République de Hollande avec les Pays-Bas méridionaux et la France. Enfin, il faut noter la localisation extrêmement périphérique des territoires hollandais et autrichiens par rapport à leurs centres politiques, La Haye et Bruxelles. Séparés respectivement des autres parties de la République de Hollande et des Pays-Bas autrichiens, ces territoires fragmentés constituaient de véritables enclaves.

5 A une époque où il n'y avait pas de caserne, cette partie de la Basse Meuse - une région fertile de polyculture où de grosses métairies, souvent fortifiées, l'emportaient sur le veld, les bois et la bruyère - était fortement convoitée comme quartiers d'hiver pour les armées $^{6}$. Une partie de cette région servait également, comme hinterland lointain de Maastricht, de grenier à la ville et à sa garnison ${ }^{7}$. Mais ces ressources suscitaient aussi le fléau des soldats démobilisés, que chaque village avait à subir ${ }^{8}$. Plusieurs industries (textiles, métaux, cuir) s'étaient développées, non seulement dans les villes, mais aussi dans les villages. La région toute entière était en quelque sorte le prolongement de l'importante concentration industrielle qui entourait Liège. Outre l'agriculture et le commerce, plusieurs manufactures domestiques faisaient ainsi vivre la population.

6 A cette géographie correspondait une formation sociale " seigneuriale "9 hautement stratifiée, comprenant une petite noblesse terrienne et un clergé, qui vivaient confortablement dans de splendides manoirs, dont le fameux monastère " Rolduc ", et contrôlaient la majorité des terres ; un groupe d'agriculteurs propriétaires de leurs terres, qui géraient aussi les métairies ; et un groupe, plus nombreux et plus diversifié, d'artisans, de manœuvres et de marchands au détail, n'ayant que peu ou pas de biens. Les tribunaux locaux et les autres services publics étaient aux mains de la noblesse et des agriculteurs. Le pouvoir des propriétaires terriens, du clergé et des agriculteurs se manifestait également dans les symboles de l'autorité et de la subordination : l'architecture de la salle d'audiences et des potences, les manoirs et les châteaux, les monastères et les métairies, dispersés et entourés de murs, témoignaient d'une hégémonie culturelle. Autant que le style de vie, le vêtement et les manières de la petite noblesse, ces maisons et ces espaces faisaient partie de la mise en scène du pouvoir aristocratique ${ }^{10}$.

Les Bandes

7 A partir de 1730 environ jusqu'à 1774 , de nombreuses églises catholiques romaines et des fermes de cette partie de la vallée orientale de la Meuse furent mises à sac lors d'expéditions nocturnes. Les victimes étaient souvent maltraitées, et quelques-unes perdirent la vie. Entre 1741 et 1778, des tribunaux locaux procédèrent, en trois étapes successives, à des arrestations en masse, suivies de procès et de condamnations à l'encontre de plus de six cents personnes (parmi lesquelles une trentaine de femmes) pour leur appartenance à des bandes et leur participation à des pillages, lesquels, qualifiés de vols, constituaient un crime capital - comme partout aux débuts de l'Europe moderne. Plus de la moitié des accusés furent condamnés à mort et exécutés publiquement " pour inspirer la peur et faire un exemple ". De nombreux autres furent bannis, la plupart in absentia. Un nombre important mourut en prison, en attente de jugement. Seuls quelques-uns bénéficièrent d'un non-lieu et furent relâchés (voir tableau 1). 
8 La biographie collective des Cavaliers du Bouc révèle que ces voleurs n'étaient pas à proprement parler des bandits, c'est-à-dire des hors-la-loi. Au contraire, tous menaient une vie apparemment ordinaire dans leurs villes respectives. La plupart d'entre eux étaient mariés, avaient des enfants et une résidence fixe. En fait, beaucoup étaient nés et avaient grandi dans la région où ils avaient lancé leurs raids - les territoires politiquement fragmentés au nord de Maastricht et d'Aix-la-Chapelle. Certains vivaient dans le même village que leurs victimes et quelques-uns étaient même leurs proches voisins. La familiarité avec les victimes peut expliquer les divers travestissements auxquels avaient recours les voleurs, qui opéraient la nuit, d'où leur surnom de " voleurs nocturnes " (nachtdieven). Nous savons que les femmes qui en faisaient partie s'habillaient en homme, tandis que les hommes utilisaient souvent des vêtements et un langage militaires. D'autres noircissaient leur visage et portaient des masques, des perruques, de fausses barbes, des bonnets et autres coiffures étrangères " afin de ne pas être reconnus " (om niet gekend te worden), comme l'expliqua un des accusés au tribunal. Rien de surprenant donc à ce que les voleurs prennent la fuite lorsque les victimes parvenaient à donner l'alarme et à obtenir l'aide de leurs voisins, comme cela se produisit en diverses occasions car, en tant que gens du cru, ils avaient de bonnes raisons de craindre d'être reconnus. Ainsi, loin d'être des hors-la-loi, des vagabonds ou des soldats démobilisés - avec lesquels on les a confondus plus d'une fois - les Cavaliers du Bouc étaient des voleurs occasionnels à identité double, dont ils dissimulaient la partie secrète derrière un masque public de villageois ordinaires et de travailleurs. Les bandes se présentaient généralement comme des compagnies de soldats démobilisés et s'organisaient en sociétés secrètes, leurs membres se dissimulant, précaution supplémentaire, derrière de multiples identités ${ }^{11}$.

9 Si l'on observe les professions de ces voleurs (que nous avons pu retrouver pour les deux-tiers d'entre eux), les artisans et les commerçants de détail (colporteurs, rouliers) sont fortement représentés. Ensemble, ils forment près de soixante pour cent des personnes arrêtées au cours des trois séries d'opérations menées contre les bandes, tandis que les fermiers et les journaliers sont à peine vingt pour cent. Ainsi, dans une zone nettement rurale, les agriculteurs étaient notablement sous-représentés chez les brigands ${ }^{12}$. Les artisans ruraux, tout particulièrement les écorcheurs, jouèrent un rôle central dans les bandes. En fait, les premières se constituèrent à partir d'un réseau très étendu d'écorcheurs originaires de plus dix endroits. Leur travail consistait à tuer les animaux malades, à débarrasser le bétail mort, à dépecer les chevaux et à nettoyer les domaines publics de toute autre dépouille corporelle. Les écorcheurs assistaient aussi le bourreau lors des séances de torture judiciaire et l'aidaient dans son travail sur l'échafaud. Ils étaient également responsables du transport des cadavres des condamnés depuis la prison jusqu'à la potence (invariablement située à la périphérie de la juridiction), où ils devaient les pendre à des chaînes ou enterrer leurs restes. Leur impureté rituelle, résultant de ce qu'ils manipulaient " une matière qui n'était pas à sa place ", les obligeait à s'installer à la lisière des villes et des villages, leur interdisait de se marier en dehors de leur groupe professionnel et rendait difficile la recherche d'un autre travail. De ce fait, les écorcheurs formaient un réseau étendu, régional et endogame $\mathrm{e}^{13}$. Confrontés aux difficultés économiques consécutives à l'après-guerre, et exclus de toute autre activité du fait de leur "impureté ", les écorcheurs purent mettre à contribution leur vaste réseau professionnel et le capital culturel acquis dans leur profession (qui, comme nous le verrons, leur donnait un sens aigu de leur rang) et s'organisèrent en bandes à travers les frontières de la Basse Meuse ${ }^{14}$. Pendant un 
certain temps, ils furent plus efficaces que les autorités, régissant des juridictions, petites et dispersées ${ }^{15}$.

10 Ce n'est pas seulement par le nombre que les écorcheurs dominèrent les premières bandes. Ils prirent aussi une part importante dans la préparation et l'organisation des raids, tandis qu'ils contrôlaient le plus souvent le partage du butin et la revente des objets volés (dont se chargeaient les receleurs juifs dans les villes plus importantes). Pour comprendre leur prééminence (qu'ils conservèrent dans les bandes ultérieures, quand leur nombre eut considérablement diminué), on doit regarder de plus près ce qu'impliquait leur profession. Les écorcheurs partageaient leur statut social inférieur, leur localisation périphérique et leur mobilité avec d'autres groupes professionnels fortement représentés dans les bandes : colporteurs, mendiants épisodiques, musiciens, bateleurs, charretiers, marchands au détail, aubergistes et soldats démobilisés. Bien qu'ayant une résidence fixe et, de ce fait, ne faisant pas partie de ces vagabonds (fahrende Leute), tous ces gens se déplaçaient beaucoup entre villes et villages, tandis que certains d'entre eux, surtout les aubergistes et les fabricants de chaussures, servaient de principaux points de contact entre les différents réseaux sociaux ${ }^{16}$. Hormis ces populations plus ou moins itinérantes (parmi lesquelles les fileurs et les tisserands, qui furent fortement représentés dans les bandes plus tardives), les écorcheurs entretenaient également des relations avec ceux dont les activités étaient liées au travail du cuir, comme les bourreliers, les fabricants de chaussures et les cordonniers. Les bandes étaient ainsi reliées par des liens professionnels, de parenté et de mariages (qui ne se limitaient pas au seul groupe des écorcheurs) et par des solidarités locales. Il est frappant de noter que la grande majorité des Cavaliers du Bouc étaient installés dans de petites localités et des hameaux situés près de bourgades plus importantes ou à leurs abords. Comme nous l'avons dit, les Cavaliers du Bouc étaient des citoyens ordinaires et, au mieux, des voleurs épisodiques. Ils avaient une résidence fixe et, de ce fait, doivent être distingués des hors-la-loi, des vagabonds et des groupes itinérants ${ }^{17}$, même si beaucoup d'entre eux étaient très mobiles. Etant donné cette mobilité et le fait qu'ils vivaient à la périphérie, ils pouvaient difficilement être soumis à des formes rigides de contrôle social. Ceci était particulièrement vrai des écorcheurs, qui pouvaient s'organiser sur des distances considérables. Pendant longtemps, ils le firent avec bien plus d'efficacité que les autorités judiciaires, attachées à de petits districts. En un sens, c'est donc le réseau régional et endogame des écorcheurs qui fournit l'infrastructure des premières bandes.

11 Les autres implications du commerce des peaux sont également importantes pour comprendre l'organisation des bandes des Cavaliers du Bouc et la violence collective qu'ils exprimèrent. En visitant les fermes à des moments inhabituels, les écorcheurs acquirent une connaissance intime de la région où ils travaillaient. Leur sens de l'orientation a dû être exceptionnel, puisqu'ils pouvaient trouver leur chemin en pleine nuit, sur un espace très vaste, pour aller à leur rendez-vous ou vers leurs objectifs et rentrer avant l'aube, chaque déplacement nécessitant un minutage précis. Plus encore que les colporteurs, les charretiers, les amuseurs, et autres itinérants, les écorcheurs avaient un prétexte pour se trouver dans nombre d'endroits et aux moments les plus inattendus. En tant que bouchers d'urgence, leur présence, n'importe où et à n'importe quel moment, ne suscitait aucun soupçon, de même le fait de transporter de lourds paquets et des colis. Le capital culturel acquis dans le commerce des peaux comprenait également des compétences dans l'utilisation de la violence et de la douleur, notamment du fait de leur rôle de bourreau auxiliaire, ainsi qu'une familiarité avec la 
mort. D'après les descriptions des raids sur les fermes et les rectorats faites par les victimes et les coupables, les écorcheurs n'hésitaient pas à employer sur leurs victimes les moyens utilisés dans leur travail sur les animaux et dans leur rôle d'assistant du bourreau.

Les éléments portant sur l'organisation sociale et spatiale des écorcheurs et de leurs alliés ne peuvent évidemment pas expliquer les raisons pour lesquelles ils se sont organisés en bandes de brigands et en sociétés secrètes, attaquant les églises et les fermes, mutilant et tuant parfois les habitants. Ces éléments ne peuvent que nous informer sur la façon dont les écorcheurs et leurs complices pouvaient opérer. Ils jettent aussi une lumière sur les rapports de force avec les autorités attachées, elles, à des juridictions de petite dimension.

13 A ce stade, nous devons considérer les aspects culturels de la violence collective, le rôle que jouaient les notions d'identité, de fierté et de sens, dans le banditisme et sa répression. Nous avons trouvé nos indices dans les aspects les plus " significatifs " des raids, dans ce que ces expéditions ont à " dire ". Il est évident que les écorcheurs et leurs divers associés rendaient d'indispensables services à la communauté. Pourtant, la population rurale et établie des fermiers (dans laquelle les autorités locales étaient majoritairement recrutées) les excluait de ses rangs. Il n'y a donc rien de surprenant si les Cavaliers du Bouc - dès leurs premières sorties, au début des années 1730, jusqu'à leurs toutes dernières, en 1774 - ont pris pour cible de leurs opérations les principaux symboles des communautés rurales : les églises et les fermes. Si la stigmatisation des écorcheurs et de leurs associés a pu leur fournir une cause, la question n'en demeure pas moins de savoir pourquoi ces bandes sont apparues autour de 1730 et pas avant.

Il est probable que les écorcheurs jouissaient en Basse Meuse d'une certaine prospérité dans les décennies autour de 1700 . Les opérations militaires qui ravagèrent la région jusqu'au début du XVIIIème siècle et la peste bovine, qui frappa plusieurs régions d'Europe occidentale entre 1713 et 1719, ont dû simultanément favoriser le commerce des peaux et infliger de grosses pertes aux fermiers. Vers 1720 , ces calamités cessèrent pour un temps, ce qui signifiait que les jours de prospérité étaient révolus pour les écorcheurs. Divers éléments attestent d'une sensible diminution de l'emploi chez les écorcheurs dans les années 1720 et 1730. Certains durent insister sur leur monopole local ; d'autres se déplaçaient constamment pour trouver du travail ; d'autres encore changèrent de lieu de résidence. En tant qu'écorcheurs, ils avaient peu de chance de trouver un emploi en dehors de leur spécialité, aussi en appelèrent-ils à l'aide de leur parenté. Les déplacements de certains d'entre eux, essentiellement des jeunes, nous donnent des indications sur la dimension de leur réseau régional de parenté. Ils révèlent également que certains d'entre eux avaient perdu leurs attaches et commençaient à adopter les caractéristiques propres à une vie de vagabonds ${ }^{18}$.

Bien que les artisans soient restés les plus nombreux, les bandes ultérieures intégrèrent des gens d'origine différente, dont les buts étaient distincts. Dans les années 1750 et 1760 , les bandes tombèrent sous la coupe d'un chirurgien local, ancien officier dans l'armée autrichienne, qui pouvait compter sur les réseaux existants des brigands et sur les services de divers aubergistes et fabricants de chaussures - professions qui avaient été, elles aussi, fortement représentées dans les premières bandes ${ }^{19}$. C'est ainsi que ces dernières, qui avaient été et continuaient d'être un assemblage de diverses sociétés secrètes, se coulèrent dans un moule militaire. Le recrutement devint une affaire sérieuse, plus important que les raids eux-mêmes qui, exceptées cinq sorties massives 
pour la seule année 1770 dans le pays de Gulick, se raréfièrent et furent à peine rentables. A ce stade, les opérations des Cavaliers du Bouc ressemblaient fort à celles des corps-francs ou des milices dans leur façon d'agir ${ }^{20}$. Quels qu'aient pu être les objectifs politiques nourris par les dirigeants pour la suite (et on ne peut exclure l'éventualité d'un mouvement sécessionniste), ils firent long feu à la suite des arrestations de masse lancées par les autorités au début de 1771, après qu'une expédition hasardeuse, qui n'avait pas été autorisée par les chefs, eut entraîné la découverte des bandes. Tout au long de l'année 1770, environ 400 personnes furent jugées par des tribunaux locaux et la plupart condamnée pour leur participation à des pillages et leur appartenance à des bandes. Une douzaine seulement fut relâchée.

Comme je l'ai suggéré plus haut, le but des expéditions des Cavaliers du Bouc n'était pas seulement le vol, ces expéditions étaient aussi la manifestation de l'hostilité particulière des brigands à l'encontre de la population rurale établie. Nous touchons ici aux aspects symboliques de la violence collective. Les victimes étaient souvent tabassées, quelque soit leur sexe ou leur âge, et certaines furent tuées ou moururent peu après les raids. Des femmes furent violées en maintes occasions et on trouve plusieurs prêtres parmi les victimes.

Le pillage des églises, particulièrement fréquent dans les premières années, alors que les écorcheurs dominaient les bandes, impliquait davantage que le vol de biens et d'argent - et pas seulement parce que des objets sacrés faisaient partie du butin. S'introduire en force dans une église était déjà, en soi, un acte à forte connotation symbolique. Dans la mesure où l'église, en tant que centre de sociabilité, était le noyau de la communauté et la " Maison de Dieu ", de telles intrusions étaient définies comme des violations majeures et, si elles s'accompagnaient de vols, étaient considérées comme des sacrilèges et punies " par le feu ", sanction qui évoque des images d'impureté et de purification, de l'église elle-même et de la communauté des croyants ${ }^{21}$. Comme Raymond Firth le rappelle, cette communauté des croyants forme un corps, le Corps du Christ, ses membres étant en communion mystique avec Lui : les élus sont unis " en une communion et une fraternité, dans le corps mystique de Ton Fils, Christ, notre Seigneur " comme l'exprime le Livre de Prière Commune ${ }^{22}$.

Mais le pillage des églises par les Cavaliers du Bouc allait au-delà de l'intrusion et du vol de vases sacrés. Les expéditions prirent la forme de ce qu'on a appelé le " contrethéâtre " du pauvre ${ }^{23}$. En plusieurs occasions, les opérations comportèrent des parodies de messe, au cours desquelles l'un des chefs jouait le rôle de " prêtre " et distribuait l'hostie à ses hommes. Ces actes impliquaient la violation d'un autre corps : le corps eucharistique du Christ, le pain consacré (et le vin) qu'on reçoit en Sainte Communion. " Toute cérémonie religieuse crée la possibilité de messe noire ", écrit Goffman ${ }^{24}$. Dans les profanations spontanées du Corps du Christ au cours d'une messe inversée, à travers la parodie et l'inversion des rites, les écorcheurs imitaient la Sainte Communion, la caricaturant et la violant, et posaient les fondations de leurs rituels d'initiation, dont le premier dut avoir lieu dès 1737. Avec l'expansion des bandes, ces éléments $\mathrm{du}$ " contre-théâtre " se perfectionnèrent en des cérémonies secrètes qui marquaient l'entrée dans une bande ${ }^{25}$. Celles-ci servaient de cadre offensif et subversif pour l'incorporation de nouveaux membres qui, en ces occasions, étaient encouragés à affronter les corps saints : images pieuses, icônes et effigies de la Vierge, des Saints et $\mathrm{du}$ Christ. Les nouveaux membres devaient prêter serment d'allégeance dans des chapelles en bordure de route et autres lieux liminaux. Les cérémonies prenaient la 
forme d'une liturgie Romaine Catholique inversée et se célébraient face à un autel improvisé avec des bougies allumées, des statuettes saintes, et des images de saints. Les novices devaient cracher sur un crucifix, le jeter au sol et le piétiner, tandis qu'ils renonçaient à Dieu et à la Sainte Mère, jurant allégeance au Diable, promettant le secret et s'engageant à voler. En certaines occasions, les cérémonies d'initiation avaient lieu autour d'une bougie allumée, placée dans la main prise sur le cadavre d'un criminel exécuté, auquel les écorcheurs, de par leur profession, avaient un accès facile. Le Diebshand, ou Diebslicht comme on l'appelait, était censé avoir des pouvoirs magiques et faciliter les cambriolages : il ouvrait les serrures, endormait les victimes, les empêchait de se réveiller ou, si elles étaient réveillées, les empêchait de parler, de bouger, etc. ${ }^{26}$.

19 Le simple geste symbolique de piétiner un crucifix et de cracher sur lui renvoyait à cet autre corps du Christ, la communauté des croyants. Ainsi les sacrilèges aidaient-ils les candidats à l'initiation à se séparer de la " société " pour devenir membres d'une sorte de contre-société. Pour les écorcheurs et plusieurs de leurs associés, cette séparation était aussi une " imitation " - faisant appel à leur capacité de mimétisme - puisqu'ils avaient déjà été exclus de la vie sociale ordinaire à cause de leur profession, leur mise à l'écart étant symbolisée par leur ségrégation spatiale. Ecrivant sur le pillage des églises aux XVIIème et XVIIIème siècles en France (dans le Lyonnais et le Beaujolais) par des bandes de vagabonds, Renaud Ferrand considère à juste titre ces vols comme une réponse à l'exclusion sociale : " L'église est un foyer de sociabilité, l'endroit où les paroissiens se rencontrent, échangent les nouvelles, etc. L'église représente tout ce de quoi sont exclus les vagabonds : une intense sociabilité, le sentiment d'appartenance à un village, peut-être aussi une dévotion ancestrale, un lieu où se reposer en paix. L'Eglise symbolise le cœur de la communauté. Les vols reflètent le désir, probablement plus inconscient que conscient, de prendre une revanche sur cette communauté où l'on n'a aucun droit "27.

20 Comme ailleurs dans les " rites de passage ", ces rencontres secrètes des Cavaliers du Bouc ont dû renforcer la cohésion sociale du réseau des brigands, accentuer les différences entre ceux-ci et les gens ordinaire ${ }^{28}$ et aussi renforcer leur audace. Elles ont commencé à l'époque où les bandes se sont mises à croître rapidement et ne pouvaient plus être liées par les seuls liens de parenté, de mariage, d'amitié, de métiers et de liens locaux, bien que ces liens aient continué à jouer un rôle significatif, en termes de structure et d'affectivité. Les voleurs insistaient sur l'" égalité " et, durant la troisième et dernière phase, sur la création imminente, par la violence, d'un " Nouveau Royaume " et d'une " Confrérie du Bonheur ".

21 Les informations sur les cérémonies d'initiation de ces " Associés du Diable (Teufelsbündner), de même que la remarquable mobilité des bandes, valurent aux brigands d'être surnommés les Bokkeryders (Bockreiter, en allemand), c'est-à-dire Cavaliers du Bouc, nom populaire qui les a désignés au début des années 1770, au moment où les derniers procès battaient leur plein, et par lequel ils ont été connus depuis. On ne trouve pas cette dénomination dans les procès-verbaux des tribunaux qui parlent de " bandits ", de " voleurs de nuit ", d'" extorqueurs ", de " membres de la fameuse bande ", etc., bien que les juges aient connu l'existence d'une confédération d'assermentés ${ }^{29}$. Enraciné dans une croyance populaire ancienne et répandue qui associe le bouc au mal, au Diable et à ses œuvres, l'usage du terme " Bokkeryders " suggère que les voleurs étaient considérés comme des anti-sociaux auxquels on 
attribuait des pouvoirs surnaturels dont celui, magique, de voler de nuit à dos d'animal vers des lieux fort éloignés pour piller et aller à leurs rendez-vous. Mais on ne sait pas, bien sûr, pour lesquels de leurs contemporains ce terme a pu avoir des connotations surtout ironiques ${ }^{30}$.

Après avoir esquissé les principaux traits du contexte dans lequel la violence collective prit forme " par le bas ", il est tentant de réfléchir brièvement sur le penchant subversif à prêter serment par des formules sacramentelles, des " serments sacrilèges " comme les appelaient les autorités judiciaires, parce qu'il montre combien les traditions populaires et celles des élites étaient proches et comment les deux formes de violence collective étaient apparentées. La prestation de serment, point focal des rituels d'initiation de toutes les sociétés secrètes, constituait l'essentiel de la cérémonie chez les Cavaliers du Bouc. Il suggère des similarités avec les preuves d'allégeance et d'intégration (auxquelles il comportait des références) à quatre institutions majeures : la communion solennelle, l'enrôlement dans l'armée, le recrutement dans le schutten local (ou garde civile) et l'installation de nouveaux membres dans le tribunal local. Ces cérémonies d'introduction étaient imitées et en même temps, comme pour les institutions, parodiées et subverties. Comme Sigmund Freud l'écrit dans son étude sur la plaisanterie : " si la représentation par son contraire est l'une des techniques de la plaisanterie, nous pouvons nous attendre à ce que la plaisanterie puisse elle aussi utiliser son contraire - la représentation par quelque chose de similaire ou de proche "31. De façon significative, les principaux lieux où se tenaient les cérémonies de prestation de serment tout au long des années 1750 et 1760, quand les adhésions étaient en plein essor, ont été les chapelles des bords de route. L'une d'elles, la chapelle St. Léonardus, était située sur une colline du même nom, près du monastère " Rolduc ". Durant la répression des bandes au début des années 1770, les autorités exigèrent un lieu supplémentaire pour y installer de nouveaux gibets et choisirent l'emplacement de cette chapelle profanée qu'ils firent démolir à cet effet. Ces déplacements et ces substitutions impliquaient une mimésis à deux degrés des atteintes contre les corps et confirmaient l'idée qu'il y avait un dialogue, ou une " circulation ", entre la culture populaire et celle des élites ${ }^{32}$. Ceci nous amène à une deuxième forme de violence collective : les moyens dont disposaient les autorités pour réprimer le banditisme dans les territoires fragmentés de la Basse Meuse.

La Répression

Pendant longtemps, les tribunaux locaux, chargés de poursuivre les criminels dans des juridictions relativement petites, ignorèrent tout des véritables auteurs des pillages des églises et des fermes. Les magistrats croyaient en effet que ces délits étaient le fait de groupes de vagabonds. Tout ce qu'ils pouvaient faire, en l'absence d'une force de police régionale, était de renforcer ce qu'on appelait les décrets (plakkaten) contre ceux-ci et d'insister sur la vigilance de la garde civile locale ou schutten. Il est fort révélateur que, dans chacune des trois grandes opérations menées contre les Cavaliers du Bouc, les tribunaux locaux ne se mirent à coopérer (échanges d'informations, livraison de prisonniers) qu'après les premières arrestations des membres de ces bandes. Il est également significatif que les procureurs locaux aient dépendu des " erreurs " commises par les membres de la bande qui avaient fait des expéditions risquées sans autorisation de leurs chefs ou qui avaient été reconnus par leurs victimes (comme ce fut le cas en 1741, 1750, 1770, 1773 et 1774). 

étaient liés par des liens de parenté et de mariage, ce qui facilitait leur coopération. Cependant, en l'absence d'une force de police régulière et de maisons de redressement, les tribunaux n'avaient pas les moyens de réprimer les bandes autrement que par la violence, la terreur et la diffamation. Ceci est explicitement spécifié dans les attendus des sentences de mort, tot afschrik en exempel (mort pour l'exemple), et dans les stipulations additionnelles selon lesquelles le corps du condamné ne devrait pas avoir d'enterrement chrétien. Ne serait-ce que pour ces raisons, on aurait tort de considérer les pendaisons - il y en eut plus de trois cents entre 1741 et 1778 - uniquement en termes pragmatiques, c'est-à-dire comme de simples éliminations. Une telle approche de la violence collective " par le haut " passerait à côté de l'aspect le plus important de la loi criminelle sous l'ancien régime : le refus d'enterrer, qui ajoutait l'infamie à la mort $^{33}$.

Dans tous les cas enregistrés de pendaison, le corps du condamné était suspendu par des chaînes pour servir de nourriture aux oiseaux jusqu'à sa complète décomposition et sa pourriture (totdat het geheel vergaan was) ainsi que la sentence l'annonçait régulièrement lorsqu'il s'agissait d'une peine capitale. Par leur refus d'un enterrement en terre consacrée (een ordentelijke begrafenis) et son spectaculaire déplacement du centre (l'église) vers la périphérie (veld, maquis, lande), ces châtiments représentaient des inversions majeures. Ces agressions extrêmes et publiques du corps infligeaient les derniers outrages à l'honneur et au statut d'une personne, ainsi qu'à la réputation de sa famille et de ses descendants.

Les images du corps profané des condamnés jouèrent un rôle important au cours de la répression des bandes qui, on le sait, se fit en trois étapes puisqu'elles réapparurent deux fois. Il est impossible de traiter ce thème, d'une richesse considérable, dans le cadre de cet article. Nous passerons les étapes concernant l'arrestation, la détention et les rituels de la torture judiciaire, qui constituaient de sérieuses infractions à l'honneur et au statut d'une personne par le biais des atteintes contre son corps, pour nous intéresser surtout aux aspects rituels et symboliques des condamnations et de leur exécution. Le réalisme magique de la répression n'était pas moins frappant quand l'accusé était absent ou fugitif (fugitief) et jugé in absentia. Dans la plupart des cas, ces personnes étaient bannies pour toujours (voor eeuwig), des territoires hollandais ou autrichiens, et la sentence notifiait qu'ils seraient passibles de mort s'ils revenaient. Cette sentence était lue en public, puis placardée sur le gibet. Mais, dans au moins quatorze cas où l'accusé, ignorant les citations du tribunal, n'était pas parti en exil, c'est l'effigie du condamné qui fut pendue. Un simple bannissement ne suffisait pas, et la personne condamnée - par le biais d'une effigie, d'une poupée, d'une imitation de son corps - devait être magiquement retirée de la communauté. Le même produit le même, le même agit sur le même ${ }^{34}$.

Les atteintes à l'identité sociale des condamnés sont également évidentes dans trois cas enregistrés, où une peine concomitante ordonne la démolition de leur maison avec interdiction de construire sur cet emplacement pendant une période de cent ans, "à cause des horribles crimes commis par son propriétaire et parce qu'elle avait servi d'abri et de lieu de rendez-vous aux voleurs et aux fripons (schelmen) ". Les atteintes aux lieux (maison, foyer), en tant que lieu de mémoire par excellence, et le fait de transformer, de façon posthume, le condamné en sans abri, nous donne un autre 
exemple de purification rituelle, de tentative pour retirer magiquement une personne de la communauté et, aussi, de la mémoire sociale ${ }^{35}$.

Un aspect important de la violence collective infligée aux Cavaliers du Bouc concerne sa topographie : l'espace de la violence et de la mort. Situé aux limites des juridictions, le lieu de l'exécution était un emplacement clairement délimité, en général une hauteur ou une colline ( $d$ 'où les termes de galgeheuvel, galgeberg, toponymes qu'on trouve encore aujourd'hui dans cette région). Le condamné devait être escorté depuis cet autre lieu de violence qu'était le lieu de détention, habituellement les sous-sols de l'un des châteaux ou des manoirs, vers les faubourgs de sa propre ville. Ces processions jouaient un rôle important dans la chorégraphie du châtiment, puisqu'elles renforçaient l'humiliation et la disgrâce. Chaque peine reproduisait un élément du crime qui avait été commis. A juste titre, Foucault note que le corps du condamné était un élément essentiel de la cérémonie dans les exécutions publiques sous l'Ancien Régime : " C'était le rôle du coupable de porter ouvertement sa condamnation et la vérité du crime qu'il avait commis. Son corps, exposé, exhibé dans une procession, torturé, servait publiquement de support à une procédure qui était restée jusque là dans l'ombre ; en lui, sur lui, la sentence devait être lisible par tous " ${ }^{36}$. Les principaux éléments de ce code comportaient quatre formes de châtiments : le bannissement (après que le condamné ait été fouetté et parfois marqué au fer rouge), la potence, la roue, et le feu ${ }^{37}$. Les voleurs ordinaires étaient fouettés et bannis ; ceux qui avaient commis des vols qualifiés étaient pendus ; les meurtriers et les chefs de bandes étaient roués; et ceux qui avaient provoqué volontairement un incendie ou commis un sacrilège (par exemple piller des églises) étaient punis par le feu de diverses façons. Dans certains cas, des éléments du crime étaient littéralement reproduits dans la peine. De telles reproductions avaient lieu au moment de l'exécution d'un condamné qui s'était noirci le visage pour s'emparer d'une ferme, ou avait juré sur le diable. Une brochure allemande publiée en 1744 raconte : En même temps, comme souvent dans la vie / Leurs visages seront noircis / Pour qu'ils soient reconnus pour ce qu'ils sont / Avant qu'ils ne soient exécutés. / On brûle deux doigts à celui / Qui a fait le serment / De ne reconnaître que le Diable / Et qui sera puni à Neustadt ${ }^{38}$.

Dans son commentaire des ordonnances 11 et 125 du Carolina (1532), qui traite des châtiments pour incendies volontaires et contrefaçons, Radbruch note : " celui qui a péché par le feu, l'incendiaire, le faux monnayeur, doit périr par le feu " ${ }^{39}$. La violence collective que déployaient les autorités pour réprimer le banditisme faisait appel à la terreur et à l'infamie. Aussi les éléments les plus importants des châtiments incluaientils la désintégration du corps et la privation de rites funéraires. Ce qu'infligeaient les tribunaux aux condamnés était pire que la mort.

La sensibilité populaire concernant l'intégrité du corps et les soins post-mortem ressort clairement des tentatives d'intervention dans le système judiciaire. Nous connaissons des tentatives nocturnes pour enlever de la potence les restes d'un parent et lui offrir un enterrement décent. Découvertes, ces tentatives étaient considérées comme un vol et punies comme tel. Pour ceux qui étaient morts en détention, les autorités avaient en réserve, dans la plupart des cas, des peines non moins infamantes. Un arrêt était prononcé sur le corps que l'écorcheur devait traîner sur une claie jusqu'au gibet pour qu'il y soit brûlé ou pendu jusqu'à ce qu'il pourrisse, selon l'état des restes. Les suicidés étaient généralement pendus par un pied au gibet, ce que l'on peut interpréter comme la reproduction d'une inversion. Nous connaissons le cas d'une veuve dont le mari, 
mort en détention sans confession, avait été enterré sous le gibet. Elle écrivit une requête aux Etats généraux à La Haye, dans laquelle elle demandait que soit révisée la condamnation infamante, se référant explicitement au rôle de l'écorcheur et demandant que son mari fut enterré chrétiennement. Sa requête fut rejetée. Avant de prononcer sa sentence concernant le corps, la cour avait, selon une procédure classique, demandé l'avis d'un juriste de Maastricht. Le onpartijdige rechtsgeleerde avait suggéré que le corps fut rendu aux siens et " enterré de noctu, sans hommage, mais aussi sans offense par le service qui employait l'exécuteur et l'écorcheur ".

31 L'image du corps en train de se décomposer est d'un intérêt considérable - et pas uniquement dans le contexte de la répression des bandes de pillards accusées de vol et de sacrilège. Le corps décomposé, corrompu, constitue une puissante métaphore. Comme Bruce Lincoln l'a mis en avant, dans un contexte différent mais très similaire (l'exhumation notoire et l'exposition publique de corps de prêtres, de nonnes et de saints depuis longtemps enterrés dans plusieurs villes et cités, en Espagne pendant la Guerre civile), l'image d'un corps en décomposition fournit une métaphore de la corruption morale : " comme ses presque synonymes pourriture et décadence, le mot corruption est parfaitement concret et tout-à-fait manifeste dans le cas d'un corps en décomposition "40. Comme le suggère Lincoln, la corruption corporelle, d'un point de vue théologique, " est un processus moral aussi bien que naturel, car la décrépitude est le résultat physique ultime d'une vie de péchés, c'est-à-dire d'une vie corrompue. Et, qui plus est, les corps de ceux qui ont été purifiés de tout péché par les sacrements de l'Eglise et la pratique d'une vie de sainteté ne pourrissent pas et s'approchent de l'éternité, la non-décomposition étant l'une des preuves majeures de sainteté "41.

Conclusion

Bien que, dans une grande mesure, la culture de l'élite et la culture populaire en Basse Meuse aient constitué des modèles l'une pour l'autre et l'une de l'autre, il serait pourtant certainement exagéré de dire qu'elles se sont développées dans une relation mutuellement constitutive. Mais il y avait une bonne part de " reflet " - imitation, mime, parodie - de la part des groupes subalternes, tandis que la violence des châtiments reflétait quelque chose de l'échelle, de l'ampleur, de la forme et du sens des crimes, réels et imaginaires. La violence massive que les deux groupes s'infligeaient réciproquement (sans précédent dans l'histoire de la République hollandaise et étrangement négligée dans l'historiographie officielle hollandaise) et son réalisme explicitement magique ne peuvent être compris que si l'on se réfère aux caractéristiques les plus marquantes de ces territoires : leur fragmentation politique, leur localisation périphérique, et leur structure seigneuriale qui faisaient se côtoyer splendeur aristocratique et misère plébéienne. Ces conditions fournissaient à l'évidence un espace considérable aux groupes dissidents ${ }^{42}$, mais provoquaient la répression et définissaient ses modalités. Confrontés à des formes massives et prolongées de subversion, les membres de la classe dirigeante restauraient leur domination par le biais du théâtre de la loi, étouffant par la même occasion les voix contestataires. Le tribunal (salle d'audience), le lieu de détention, la rue et le lieu de l'exécution fournissaient le cadre de cette affirmation d'hégémonie culturelle, permettant, tout en introduisant un élément dramatique, de mettre l'accent sur la distinction entre le corps intègre d'une part, et le corps violé, déshonoré, pourrissant d'autre part - distinction qui, par un jeu métonymique (pars pro toto), permettait une restauration magique de l'ordre. 
Tableau 1 :

\begin{tabular}{|l|c|c|c|c|}
\hline SENTENCES & I & II & III & Total \\
\hline $\begin{array}{l}\text { Sentences de mort non précisées, mais } \\
\text { probablement le gibet }\end{array}$ & 5 & & 45 & 50 \\
\hline Gibet & 45 & 16 & 191 & 252 \\
\hline Gibet avec supplices & 1 & 1 & 1 & 3 \\
\hline Supplice de la roue & & 1 & 4 & 5 \\
\hline Idem avec supplices additiomnels & 5 & & 1 & 6 \\
\hline Garrot & & & 2 & 2 \\
\hline Idem avec supplices additiomels & 27 & & & 27 \\
\hline Décapitation & 6 & & & 6 \\
\hline Idem avec supplices additiommels & 4 & & & 4 \\
\hline Morts en détention & 12 & 4 & 31 & 47 \\
\hline $\begin{array}{l}\text { Echappé de la détention après la } \\
\text { condamnation à mort }\end{array}$ & & & 3 & 3 \\
\hline Baunissement & & & 2 & 2 \\
\hline Idem avec supplices & 1 & & 9 & 10 \\
\hline Condammation in abstentia & 1 & & 58 & 59 \\
\hline Bannissement avec pendaison en effigie & 1 & 5 & 8 & 14 \\
\hline Réclusion & 5 & & 3 & 3 \\
\hline Élargissement & 114 & 27 & 371 & 512 \\
\hline Relâché faute de preuve & & 12 & 17 \\
\hline Total & & & 1 & 2 \\
\hline
\end{tabular}

Tableau 2:

\begin{tabular}{|l|c|c|c|c|}
\hline \multicolumn{1}{|c|}{ Professions } & I & II & III & TOTAL \\
\hline Artisans & 62 & 15 & 142 & 219 \\
\hline Commerce/ transports & 21 & 5 & 33 & 59 \\
\hline Agriculteurs & 10 & 5 & 73 & 88 \\
\hline Autorités & 3 & 3 & 11 & 17 \\
\hline Aubergistes & 5 & & 10 & 15 \\
\hline Autres & 22 & 2 & 19 & 43 \\
\hline Total & 122 & 30 & 288 & 441 \\
\hline
\end{tabular}

33 * on dispose de la profession d'environ deux-tiers des condamnés. A peine plus de 24 d'entre eux avaient une expérience militaire.

\section{NOTES}

1.Tilly Charles, " Anthropology, history, and the Annales ", Review, 1, 3/4, 1978. 
2.Wallerstein Immanuel, The modern world-system, New York, Academic Press, 1974, p. 141.

3.Pour plus de détails, voir Blok Anton, De Bokkerijders, Roversbenden engeheime genootschappen inde Landen van Overmaas, 1730-1778, Amsterdam, Prometheus, 1991. 4.Sur l'histoire militaire et politique de la Basse Meuse, cf. Wouters H. H. E., Grensland en bruggehoofd. Historische studies met betrekking tot het Limburgse Maasdal en, meer in het bijzonder, de stad Maastricht, Assen, Van Gorcum, 1970. Haas J.A.K., De verdeling van de Landen van Overmaas, 1644-1662, Assen,Van Gorcum, 1978. Gutmann Myron P., War and rural life in the early modern Low Countries, Princeton, Princeton University Press, 1980.

5.Childs John, Armies and warfare in Europe, 1648-1789, Manchester, Manchester University Press, 1982, p. 102-103. Pour plus de détails sur les sièges de Maastricht entre 1620 et 1750 (soit en 1632, 1673, 1674, 1676, 1703 et 1748) voir Gutmann Myron P, op. cit., p. 16, 22, et passim. Voir aussi Thewissen M. A. F., " Limburg van 1648 tot 1795 ". Limburgs verleden, II, p. 302-55. Maastricht, 1967 ; Wouters H. H. E., op. cit. 6.Sur le cantonnement des troupes et le développement tardif des casernes, cf. Child John, Armies and warfare in Europe, 1648-1789, Manchester, Manchester University Press, 1982, p. 174 et sq. Sur les quartiers d'hiver en Basse Meuse, cf. Wouters H. H. E., op. cit., p. 229. Sur la fertilité de la région, sa démographie, son peuplement, cf. Hekker Hekker R. C., " De dorpen ", in R.C. Hekker et al. (eds.), Dorp en stad in Limburg, Zutphen, Walburg Pers., 1981 ; Renes J., Degeschiedenis van het zuidlimburgse cultuurlandschap, Assen, Van Gorcum, 1988, p. 107-69 ; Hillegers H. P. M., Heerdgang in zuidelijkLimburg, Maastricht, Natuurhistorische Genootschap in Limburg, 1993, p. 52-63.

7.Cf. Wouters H. H. E., op. cit., p. 395 ; Haas J.A.K., op. cit., p. 234-35.

8.Cf. Wouters H. H. E., op. cit., p. 324.

9.Pour la caractérisation du terme "seigneurial" (en hollandais: overherigheid) appliqué à cette partie de la Basse Meuse, cf. Wichers (1965 : 198-99, 234 et passim). 10.Sur l'importance de la petite noblesse en Basse Meuse, cf. Jongen (1961); Wouters H. H. E., op. cit, p. 325-328 ; Haas J.A.K., op. cit., p. 202-06, 234-36 ; Janssen de Limpens (1982) ; Corten (1984). Je m'inspire aussi de E.P.Thompson, qui écrit : " les styles employés dans la politique et l'architecture, la rhétorique de la petite noblesse et ses arts décoratifs, tout semblait proclamer la stabilité, la confiance en soi, l'habitude de maîtriser n'importe quelle menace contre leur hégémonie... le contrôle par cette classe dirigeante du XVIIIème siècle résidait d'abord et avant tout dans son hégémonie culturelle, et secondairement dans l'expression d'un pouvoir économique ou physique (militaire). Dire que c'est culturel ne signifie pas pour autant que c'est immatériel, trop fragile pour une analyse, inconsistant. Définir le contrôle en termes d'hégémonie culturelle ce n'est pas abandonner toute tentative d'analyse, c'est au contraire se mettre en état de faire l'analyse sur le terrain où elle doit être faite : dans les représentations du pouvoir et de l'autorité et les mentalités populaires de subordination ", cf. Thompson E. P., " Patrician society, plebeian culture ", Journal of Social History, 7, 1974, p. 387. Comparer avec Thompson E. P., " Folklore, anthroplogy, and social history " Indian Historical Review, 3, 1978, p. 254.

11.L'importance fondamentale du secret dans la vie sociale a depuis longtemps été reconnue par Simmel, qui considérait le secret, fait de cacher la vérité par des moyens positifs ou négatifs, comme l'une des plus grandes réalisations humaines, cf. Simmel Georg, The secret society. The sociology of Georg Simmel, Tr. Kurt H. Wolff. Glencoe, 
Ill., The Free Press., 1950, p. 330 (1ère ed. 1908). A propos des sociétés secrètes, il remarquait que : " De toutes les mesures de protection, la plus radicale est de se rendre invisible. A cet égard, les sociétés secrètes se différencient radicalement de l'individu qui cherche la protection du secret. L'individu ne peut le faire efficacement que pour des entreprises ou des situations particulières ; d'une façon générale, il peut incontestablement se cacher pendant un certain temps, mais son existence, hormis quelques combinaisons très abstruses, ne peut pas en soi être un secret. Alors que la chose est tout à fait possible pour un petit groupe social. Ses membres peuvent vivre dans des interactions très fréquentes, mais le fait qu'ils forment une société, une conspiration ou une bande d'escrocs (eine Gaunerbande), un conventicule religieux ou une association s'adonnant à des orgies sexuelles, peut, par essence et de façon permanente, être un secret ", ibidem, p. 345-46.

12. Voir tableau 2

13.Dans certaines régions d'Europe, les peaussiers restèrent socialement des horscastes jusqu'à la fin du XIXème siècle. Dans son Volkskunde der Schweiz, (Folklore de Suisse) Weiss écrit : " l'écorcheur, ou l'écarisseur, mène encore dans de nombreux villages une existence de proscrit à la périphérie des villages dans sa cabane d'écorcheur.A Schanfigg, on se souvient encore du temps où l'écorcheur devait descendre sur le bas-côté de la route quand il croisait quelqu'un, et où on ne lui donnait à manger que s'il avait emporté avec lui son propre couvert ", cf. Weiss Richard, Volkskunde der Schweiz. Grundriss, Erlenbach-Zizrich, Eugen Rentsch Verlag, 1946, p. 113.

14.Les guerres et autres opérations militaires, même une simple présence militaire, fournissaient du travail, non seulement à ceux qui y participaient, mais aussi à ceux dont les services étaient nécessaires pour soutenir les activités militaires. Parmi ces derniers, on trouve les écorcheurs, les tanneurs, les bouchers, les fabricants de chaussures, les bourreliers, les forgerons et autres serruriers. Les vicissitudes des Eta au Japon, groupe professionnel méprisé, spécialisé dans la boucherie, le tannage et le travail du cuir (ce qui dans la société bouddhiste est considéré comme une souillure) étaient à cet égard très similaires à celles des peaussiers en Basse Meuse. Ils étaient prospères en période de guerre civile quand leurs services étaient fortement requis. Mais les Eta souffrirent de la paix relative qui régna sous les Tokugawa (1603-1868) quand leurs commerces perdirent de l'importance qu'ils avaient eue aux temps de la guerre civile, tandis qu'ils étaient de plus en plus discriminés, cf. Price John, " A history of the outcaste : Untouchability in Japan ", in George De Vos \& Hiroshi Wagatsuma (eds), Japan's invisible race, Berkeley/Los Angeles, University of California Press, 1972. 15.Dans la poursuite de ces bandes (1743-4, 1750-52, 1770-78), les cours locales coopérèrent ensemble (échangeant des informations, ramenant les fugitifs); elles purent le faire efficacement parce que les principaux magistrats de ces cours, particulièrement les schouten et les drossaarden (baillis) appartenaient au réseau régional de la gentry (voir diagramme 1).

16.Sur le rôle subversif des tavernes et des auberges, voir Scott James C., Domination and the arts of resistance. Hidden transcripts, New Haven, Yale University Press, 1990, p. 121-122, qui écrit : " Ici, les classes inférieures se rencontrent, loin des traiteaux et de leur activité habituelle, dans une atmosphère de liberté encouragée par l'alcool ". Sur le rôle central des cabaretiers dans la Bande d'Orgères voir Richard Cobb, Reactions to the French Revolution, London, Oxford University Press, 1972, p. 191. Pour l'influence des aubergistes dans la vie villageoise hollandaise, voir Wichers A.J., De oude 
plattelandsbeschaving. Een sociologische bewustwording van de " overherigheid ". Assen, Van Gorcum, 1965, p. 37. Les aubergistes avaient par définition affaire à des étrangers et des gens d'ailleurs. Ce qui affectait leur réputation dans ces communautés rurales relativement fermées où tout étranger est habituellement vu avec suspicion. 17.Le problème du vagabondage en Basse Meuse a été discuté par Camps Rob, " Vagebonden in Venlo 1713-1794 ", Publications, 124, 1988, p. 201-63. Sur les groupes itinérants, voir Berland Joseph A., No five fingers are alike. Cognitive amplifiers in social context, Cambridge, Mass., Harvard University Press, 1982 ; Rao Aparna (ed.), The other nomads. Peripatetic minorities in cross-cultural perspective, Köln/Wien, Böhlau Verlag, 1987.

18.Comparer avec les observations de Wilbertz Gisela, Scharfrichter und Abdecker im Hochstift Osnabrück, Osnabrück, 1979, p. 157-58 ; et de Küther Carsten, Menschenauf der Strasse. Vagierende Unterschichten in Bayern, Franken und Schwaben in der zweiten Hälfte des 18, Jahrhunderts, Göttingen, Vanden Hoeck \& Ruprecht, 1983, p. 53-54, sur le sort des écorcheurs des territoires allemands.

19.Dans la France du XVIIIème siècle (comme ailleurs), les chirurgiens étaient les praticiens de la médecine auxquels on s'adressait le plus habituellement et étaient appelés " les médecins des pauvres ". Le développement de leur profession avait été stimulé par l'avènement d'une armée de métier au XVIIème siècle, cf. Wellman Kathleen, La Mettrie. Medicine, philosophy, and Enlightenment, Durham/London, Duke University Press, 1992, p. 16-17, 29. Sur le rôle essentiel des fabriquants de chaussures dans les mouvements populaires des débuts de l'Europe moderne, voir les travaux de Hobsbawm et Scott (1980) qui en énumèrent les raisons : leur travail facilitait chez eux la reflexion et l'apprentissage de compétences telles la lecture et l'écriture, leurs échoppes fonctionnaient comme des lieux de rencontre (tout comme les auberges); parmi leurs clients il y avait des gens de toute sorte ; et les fabriquants de chaussures faisaient partie des rares personnes qui étaient lettrées et en contact régulier avec le monde extérieur...ainsi pouvaient-ils canaliser le mécontentement populaire. Il faut aussi reconnaître que la position sociale inférieure des fabriquants de chaussures provenait d'autres aspects de leur commerce (tout particulièrement quand ils étaient amenés à réparer plutôt qu'à fabriquer des chaussures) : ils travaillaient le cuir; ils étaient en contact avec les pieds (et le sol), et ils réparaient des choses qui avaient été en contact avec le corps humain.

20.Sur la genèse des corps-francs dans les territoires allemands au milieu du XVIIIème siècle, voir Childs John, op. cit., p. 119.

21.Le Carolina ( $1532: 172$ et sq.) spécifie les peines encourues pour ces formes de sacrilèges. Pour des cas similaires de vols dans des églises, en France, au XVIIIème siècle, et pour des peines similaires, voir Ferrand Renaud, " Le vol dans les églises en Lyonnais et en Beaujolais (1679-1789) : le sacrilège des exclus ", Bulletin du Centre d'histoire économique et sociale de la région lyonnaise, 2, 1989, p. 65 ; et Gutton JeanPierre, La société et les pauvres. L'exemple de la généralité de Lyon 1534-1789, Paris, Les Belles Lettres, 1971 p. 196-221.

22.Dans ses remarques sur le symbolisme du corps, Raymond Firth distingue trois corps du Christ : le corps physique, le corps mystique et le corps eucharistique, cf. Firth Raymond, Symbols public and private, London, Allen \& Unwin, 1973, p. 227. 23.Thompson E. P., " Folklore, anthroplogy, and social history ", Indian Historical Review, 3, 1978, p. 254). Voir plus loin.

24.Erving Goffman, Interaction ritual, New York, Pantheon Books, 1967, p. 86. 
25.La présence d'éléments de protestation et de parodie dans la façon dont le pillage réussi d'une église était célébré ne fait aucun doute. On peut considérer cela comme un exemple de ce que E. P. Thompson décrit comme le " contre-théâtre " de groupes subalternes : " dans toutes les sociétés, bien sûr, le théâtre est une composante essentielle tant du contrôle politique que de la protestation, voire de la rébellion. Les dirigeants mettent en scène la majesté, la superstition, le pouvoir, la richesse, la justice sublime; le pauvre fait du contre-théâtre, occupant les estrades des marchés dans les rues et employant le symbolisme du ridicule ou de la protestation ", cf. " Folklore, anthroplogy, and social history ", Indian Historical Review, 3, 1978, p. 254. Voir aussi Thompson E. P., " Patrician society, plebeian culture ", Journal of Social History, 7, 1974, p. 387 et sq. Pour une documentation exaustive sur les formes plus cachées de contestation et de résistance, voir Scott James C., op. cit., 136 et sq.

26.Cette croyance semble avoir été commune, aux débuts de l'Europe moderne.

Danckert écrit : " celui qui coupe la main d'un voleur encore pendu à la potence, y place une bougie allumée et la pose, s'assure de la réussite d'un vol ", cf. Danckert Werner, Unehrliche leute. Die verfehmten Berufe, Bern/Muenchen, Francke Verlag, 1963, p. 42.Voir aussi Bächtold-Stäubli Hanns (ed.), " Dieb Diebstahl " in Handwörterbuch des deutschen Aberglauben, Vol. II. Berlin/Leipzig, Walter de Gruyter Verlag, 1929/30, p. 229-31. Sur le pouvoir bénéfique du Diebsdaum, voir Angstmann Else, Der Henker in der Volksmeinung. Seine Namen und sein Vorkommen in dez mündlichen Volksueberlieferung, Bonn, Fritz Klopp Verlag, 1928, p. 93-94. Pour un récit plus général sur le pouvoir magique de matériel liminal, voir Leach, E. R., " Anthropological aspects of language : Animal categories and verbal abuse ", in E. H. Lenneberg (ed.), New directions in the study of language, Cambridge, Mass., Cambridge University Press, 1964 ; Leach, E. R, Culture and communication, Cambridge, Cambridge University Press, 1976.

27.Ferrand Renaud, op. cit., p. 71-73. Voir aussi Gutton Jean-Pierre, op. cit., sur le brigandage à la même période, dans la même région.

28.La Fontaine écrit : " Le rituel crée les frontières qui séparent les membres des nonmembres car il met théâtralement l'accent sur la distance qui sépare les deux statuts entre lesquels les postulants doivent passer. Les rituels d'initiation sont presque toujours irréversibles ; un initié ne peut inverser le processus et redevenir un noninitié. Le savoir une fois acquis ne peut être non-appris et puisque les sociétés secrètes sont organisées autour d'une connaissance secrète, il est compréhensible qu'elles ne peuvent admettre des démissions ". Cf. La Fontaine Jean, Initiation, Manchester, Manchester University Press, 1985, p. 58, 72, 73).

29.Il y avait longtemps que les tribunaux d'Europe occidentale avaient perdu tout intérêt dans la démonologie, cf. Levack Brian P., The witch-hunt in early modern Europe, London/New York, Longman, 1987, p. 170 et sq.). C'était particulièrement le cas dans les Basses Terres qui n'ont connu que peu de procès en sorcellerie et qui de ce fait perdirent tout intérêt pour le sujet bien avant qu'il parvienne à son apogée dans certains des pays voisins. Les quelques phrases, en Basse Meuse, qui font référence explicitement à des serments exigés emploient pour désigner cette impiété invariablement des termes comme " blasphème ", ou " sacrilège ", c'est-à-dire de godslasterlijke eed.

30.Deux rapports contemporains font référence à la dénomination de " Bokkeryders ", mais se contentent d'enregistrer l'usage et la croyance populaires qu'on y associait, tout en exprimant des réserves personnelles. Voir Mengels Winand, " Memorie of 
Kronyckboek ", Publications, 24, 1887, p. 269 ; et Sleinada S. J. P., Oorsprong, oorzaeke, bewys en ondekkinge van een godlooze bezwoorne bende nachtdieven en knevelaers binnen de Landen van Overmaeze en aenpaelende landtstreeken ontdekt, Maastricht, Reprint 1972, p. 61-62 (1ère ed. 1779). Bien entendu, on ne peut exclure que les rituels d'initiation des voleurs et le terme de Bokkeryders se référaient à des représentations courantes lors des procès en sorcellerie, aux XVIème et XVIIème siècles. Cependant, ces images, en particulier le vol magique sur des animaux et l'association entre le mal et les boucs, appartenaient à un substrat plus ancien de croyances populaires, cf. Blok Anton, " Rams and billy goats. A key to the Mediterranean code of honour ", Man (N.S.), 16, 1981, p. 427-40. La notion de " bouc émissaire ", mieux rendue par le terme allemand Sündenbock, figure originellement dans le Lévitique (16:1-34). Matthieu (25:31-45) décrit la fameuse scène où Jésus sépare les brebis des boucs, c'est-à-dire les bons des méchants.

31.Freud Sigmund, Jokes and their relation to the unconscious, Tr. James Strachey, New York/London, Norton, 1963, p. 74 (1ère ed. 1905).

32.Voir Luria Keith, Romulo Gandolfo, " Carlo Ginzburg : An interview ", Radical History Review, 35, 1986, p. 108.

33.Pour une large documentation sur les sensibilités de l'époque concernant l'intégrité des cadavres, voir Linebaugh Peter, " The Tyburn Riot against the surgeons " in Douglas Hay and al (eds.)., Albion's fatal tree. Crime and society in eighteenth-century England, New York, Pantheon Books, 1975. Linebaugh Peter, The London hanged. Crime and civil society in the eighteenth century, Cambridge, Cambridge University Press, 1992, p. 74 et sq ; Rupp Jan C.C., " Michel Foucault, body politics and the rise and expansion of modern anatomy ", Journal of Historical Sociology, 5, 1992, p. 31-60 ; et particulièrement Richardson Ruth, Death, dissection and the destitute, London, Routledge, 1987, qui affirme que de tels châtiments (qui mettent l'accent sur la désintégration du corps et le refus d'enterrer) " n'auraient comporté aucune signification culturelle (sans) un consensus attachant une grande importance aux soins post-mortem et à l'enterrement intégral du corps ", p. 28. Voir Laqueur Thomas, " Bodies, death, and pauper funerals ", Representations, 1, 1983, p. 109-31, sur les funérailles ignominieuses des pauvres et leur rapport avec l'exclusion de ces derniers du corps social aux XVIIIème et XIXème siècles en Angleterre.

34. Voir Mauss Marcel, A general theory of magic, Tr. Robert Brain, London, Routledge, 1972, 64 et sq. (1ère ed. 1903) qui discute les " trois lois de la magie " : la loi de similarité (le même produit le même), la loi de contiguïté (la partie vaut le tout, totem ex parte), et la loi d'opposition (les opposés agissent sur les opposés), et qui reconnait l'intime relation entre ces principes. Pour un exposé plus récent sur l'" analogie " et la " célébration " comme quintessence des actes magiques, voir S. J. Tambiah (ed.), Culture, thought, and social action. An anthropological perspective, Cambridge, Mass., Harvard University Press, 1985, p. 64-72.

35.Ceci pourrait être une illustration des interactions entre la culture de l'élite et la culture populaire, entre autorité et subversion. Dans certaines opérations menées par les bandes, plus particulièrement les attaques contre les auberges, le saccage comportait la destruction volontaire du mobilier, des portes, des fenêtres, des placards, des tonneaux etc..., et ont tout l'air d'avoir été des actes de vengeance. Cet aspect des raids ressemble fort aux formes plus violentes que sont les charivari ou "musique violente" (c'est-à-dire des formes de tribunal populaire) qui, dans les zones allemandes, étaient appelées Wüstung ou destruction dans le but de chasser des résidents devenus 
indésirables de la communauté. Voir Meuli Karl, " Ueber einige alte Rechtsbräuche/ Charivari ", in Karl Meuli, Gesammelte Schriften, Vol. I, Basel, Schwabe \& Co. Verlag, 1975, p. 457-59, 472-75, sur le Dachabdecken (enlèvement du toit) et le Hauswüstung (destruction des maisons).

36.Foucault Michel, Discipline and punish. The birth of the prison, Tr. Alan Sheridan. New York, Pantheon Books, 1977, p. 43. Voir aussi Gernet sur les exécutions publiques dans la Grèce ancienne : " La victime (de la peine capitale) est exposée comme un objet d'indignation publique et de rires cruels. La victime est aussi un exemple, dans la mesure où l'agonie est plus efficace qu'un corps mort " ; cf. Louis Gernet, The anthropology of Ancient Greece, Tr. John Hamilton, Baltimore, Johns Hopkins University Press, 1981, p. 267. Sur le caractère public des exécutions dans la Rome ancienne, voir Wiedemann, qui observe : " Pour que le public soit rassuré sur le fait que le bon ordre social est restauré grâce à des peines appropriées, le châtiment doit en quelque sorte être public... Les états pré-industriels ne pouvaient s'appuyer ni sur une police fiable ni sur des prisons sûres pour donner à leurs populations une telle assurance. C'est donc le châtiment lui-même qui pouvait rassurer les populations, et cela explique pourquoi les peines devaient être tout naturellement à la fois cruelles et publiques " ; cf. Wiedemann Thomas, Emperors and gladiators, London/New York, Routledge, 1992, p. 71-72.

37.Goffman discute du mot " stigmate " au sens littéral et originel. Ce terme fait référence à " des signes corporels destinés à informer par quelque chose d'inhabituel et de mal sur le statut moral de celui qui les porte. Les signes étaient découpés dans ou brûlés sur le corps et indiquaient qu'il s'agissait d'un esclave, d'un criminel, ou d'un traitre... une personne portant une tare, rituellement polluée, à éviter, spécialement dans les lieux publics ", cf. Goffman Erving, Stigma. Notes on the management of spoiled identity, Harmondsworth, Penguin Books, 1963, p.1. Sur le rachat de l'honneur par des ordalies physiques auto-infligées, voir Barton Carlin A., Savage miracles, " The redemption of lost honor in Roman society and the sacrament of the gladiator and the martyr ", Representations, 45, 1994, p. 41-71.

38.Dabey auch (wie Viel im Leben) / Wurd geschwärzt ihr Angesicht, / Um das auch an Tag zu geben / Eh' sie wurden hingericht. / Man thät die zwei Finger brennen / Dessen der geschworen hat, / Nur den Teufel zu erkennen, / Wurd gestrft auch zu Neustadt. 39.Cf. Radbruch Gustav (ed.), Die peinliche Gerichtsordnung Karls V. von 1532 (Carolina), Stuttgart, Reclam, 1960, p. 10. Dans son ouvrage Langbein John H. (Prosecuting crime in the Renaissance. England, Germany, France, Cambridge, Mass., Harvard University Press, 1974, p. 167) est d'accord avec Radbruch (Ibidem, p. 14) sur le fait que " la Carolina n'est pas vraiment un code pénal (Strafgesetzbuch) mais un code de procédure pénale (Strafprozessordnung) au sein duquel un code pénal a été inserré ".

40.Bruce Lincoln qui en donne l'étymologie : " quasi littéralement, corruptio... comme son parent espagnol corrupcion - dénote l'état de quelque chose qui est tombé en morceaux, le terme venant du latin cor-ruptio (pour con-ruptio), nom abstrait avec un sens passif, construit à partir du verbe rumpo : casser en morceaux ". Cf. Lincoln Bruce, " Revolutionary exhumations in Spain, July 1936 ", Comparative Studies in Society and History, 27, 1985, p. 257.

41.Lincoln Bruce fait remarquer que cette conception n'est pas propre à l'Eglise catholique, cf. Ibidem. Dans son livre sur castes et culture au Japon, De Vos écrit que " les vertus morales, ou la bonté, sont quasi universellement identifiés à la propreté 
physique. Il y a des chaines d'associations telles que supérieur-bon-vertueux-propreblanc-pur-inoffensif et le contraire - inférieur-mauvais-corrompu-sale-noir-souillédangereux. Des associations individuelles à l'un quelconque des termes de ces chaines peut mener à la diffusion symbolique dans les autres termes ". Cf. De Vos George, " Toward a cross-cultural psychology of caste behavior " in George De Vos \& Hiroshi Wagatsuma (eds), Japan's invisible race, Berkeley/Los Angeles, University of California Press, 1972, p. 365. Ce qui nous ramène à Marcel Mauss et ses trois " lois de la magie ". 42.Voir Scott James C., op. cit., p. 108 et sq.

\section{RÉSUMÉS}

Organised bandirty is rarely tackled by studies on collective violence, though this aspect is truly interesting because it generally simultaneously reveals two forms of collective violence. These two forms are banditry itself, and the public authority repression. Anton Blok analyses in this contribution the ascent of the goat-riders (« cavaliers du bouc »), these 18th Century riders who raged in the French Basse Meuse region, and the repression they suffered from. The author shows that even if these forms of violence were new to the region, they had relatively weak effects : the 'goat-riders' were eventually military defeated and the former social order thus reestablished. Thereby, the author shows that forms of massive violence can remain geographically and historically circumscribed. Concerning these horsemen, this circumscribtion was such that they have been forgotten by the official history.

\section{INDEX}

Mots-clés : criminalité, délinquance, répression, histoire

Index géographique : Benelux

Index chronologique : 18ème siècle

\section{AUTEUR}

\section{ANTON BLOK}

International Institute of Social History, Amsterdam School for Social Science Research. Cette communication était initialement destinée aux participants à la conférence organisée par l'International Institute of Social History, en l'honneur de Charles Tilly " Structure, identité et pouvoir : passé et avenir de l'action collective ", 2-4 Juin 1995, Amsterdam, Pays-Bas. Je dois remercier tout particulièrement James $C$. Scott pour ses très utiles commentaires de la précédente version de cette communication et pour son invitation, en 1993 à l'Université de Yale. Traduit de l'anglais par Juliette Minces. 